\title{
Preface
}

Paola F. Antonietti, Jérôme Droniou* and Robert Eymard

\section{An Eclectic View on Numerical Methods for PDEs: Presentation of the Special Issue "Advanced Numerical Methods: Recent Developments, Analysis and Applications"}

https://doi.org/10.1515/cmam-2018-0011

The design and analysis of numerical methods for partial differential equations (PDEs) is a field of constant growth. It seems that the more we develop numerical analysis for PDEs, the more we face questions and problems that require additional developments - maybe a consequence of an increased knowledge that gives the tools to tackle questions previously inaccessible and of the growing place of scientific computing in Physics and Engineering. The aspects currently taken into account when dealing with numerical schemes for PDEs include: the need for geometrical flexibility, as the meshes available for certain applications may comprise cells with complicated shapes, possibly degenerate faces or bad aspect-ratios; the capacity to handle complex models, with non-linearities, couplings, strong heterogeneities, weakly regular data or even singularities; the respect of physical properties of the model, such as conservation of energy or mass, and proper asymptotic behaviour. The above mentioned challenges are ubiquitous in practical problems arising from Engineering, Life Sciences, and Industry.

Such challenges require both the design of new schemes, and the development of a rigorous mathematical analysis of old and new schemes to ensure that they properly handle these questions with innovative tools made available by the most advanced research in mathematics - benchmarking is essential to assess the quality of a scheme, but it cannot cover all situations encountered in applications. The analysis of advanced numerical methods and their application to problems arising in the field of Engineering and Computer Science also identifies the key elements that make schemes robust and efficient, and clarifies relationships between the various methods.

These themes, among others, were at the core of two main events of the program on Numerical Methods for PDEs, organised at the Institute Henri Poincare (Paris, France) at the end of 2016 by D. A. Di Pietro, A. Ern and L. Formaggia. The first of these events was an introductory school on current topics in numerical methods for PDEs: virtual element methods [5], hybridizable discontinuous Galerkin methods [9], the gradient discretisation method [11], defective interface conditions [13], mimetic spectral methods [16], low-rank tensor methods [19], reduced basis method [15], adaptive and a posteriori finite element methods [10, 18]. The second event was a conference on "Advanced numerical methods: Recent developments, analysis, and applications". The whole IHP program included other major events and tackled many more issues around numerical PDEs, we refer to the webpage http://imag.edu.umontpellier.fr/event/ihp-quarter-on-numericalmethods-for-pdes/ for more details.

Paola F. Antonietti, MOX - Laboratory for Modeling and Scientific Computing, Dipartimento di Matematica, Politecnico di Milano, Piazza Leonardo da Vinci 32, 20133 Milano, Italy, e-mail: paola.antonietti@polimi.it

*Corresponding author: Jérôme Droniou, School of Mathematical Sciences, Monash University, Clayton, Victoria 3800, Australia, e-mail: jerome.droniou@monash.edu

Robert Eymard, Laboratoire d'Analyse et de Mathématiques Appliquées, UMR 8050, Université Paris-Est, 5 Boulevard

Descartes, Champs-sur-Marne 77454 Marne-la-Vallée Cedex 2, France, e-mail: robert.eymard@u-pem.fr 
We were the lucky organisers of this conference "Advanced numerical methods: Recent developments, analysis, and applications", which was focused on the aforementioned challenges of designing and analysing innovative numerical tools and methods to tackle modern issues in the field. This conference brought together researchers to discuss and present recent developments in the field of advanced numerical methods for the approximation of PDEs. It had eleven invited speakers, sixteen contributed talks and more than 90 participants.

The interest raised during this conference triggered the launch of this special issue, as a follow-up scientific activity that presents snapshots of state-of-the-art advances in some of the fields mentioned above. Each paper in this issue has been authored or co-authored by one of the plenary speakers. The result is an issue that covers a range of modern topics in numerical methods for elliptic, parabolic and hyperbolic PDEs.

The contributions can be broadly classified into four groups. The first comprises two articles that present general analysis frameworks for parabolic and hyperbolic models, by Gallouët [14] and Abgrall [1], respectively; the settings and results presented in these papers apply to and help create links between various numerical methods. In the second group, linear elliptic equations are considered with data or solutions that lack full regularity; boundary conditions are weakly imposed using Nitsche's technique and convergence results are obtained, using semi-analytical techniques by Baumann and Wihler [4] or generalisations of Strang's lemmas with weak notions of traces and mollifying tools by Ern and Guermond [12]. The preservation of physical or asymptotic properties is concerned by four contributions that make up the third group; Cancès, Chainais-Hillairet and Krell in [7] design a non-linear scheme to preserve, on generic meshes, the expected energy dissipation relation of a convection-diffusion model; Brenner, Çeşmelioğlu, Cui and Sung in [6] focus on the questions of eliminating spurious modes in a fluid-structure model; the issue of loss of accuracy due to poor mass conservation in the approximation of incompressible Navier-Stokes equations is covered by Ahmed, Linke and Merdon in [2]; finally, the design of well-balanced and asymptotic preserving schemes for hyperbolic systems, using local exact solutions, is the topic of the paper by Morel, Buet and Després [17]. The fourth group comprises two papers that deal with precise estimates with explicit constants that can be used to guide specific implementation choices: number of iterations in a domain-decomposition algorithm by Ali Hassan, Japhet, Kern and Vohralík [3], and mesh refinement algorithms (adaptivity) by Carstensen and Hellwig [8].

This classification should not hide the fact that each of these articles actually tackles more than one numerical concept or issue related to PDEs. Given the variety of topics covered in these contributions, we believe that many researchers interested in the numerical approximation of these equations will find something to ponder in this issue.

\section{References}

[1] R. Abgrall, Some remarks about conservation for residual distribution schemes Comput. Methods Appl. Math. 18 (2018), no. 3, 327-351.

[2] N. Ahmed, A. Linke and C. Merdon, Towards pressure-robust mixed methods for the incompressible Navier-Stokes equations, Comput. Methods Appl. Math. 18 (2018), no. 3, 353-372.

[3] S. Ali Hassan, C. Japhet, M. Kern and M. Vohralík, A posteriori stopping criteria for optimized Schwarz domain decomposition algorithms in mixed formulations, Comput. Methods Appl. Math. 18 (2018), no. 3, 495-519.

[4] R. Baumann and T. P. Wihler, A Nitsche finite element approach for elliptic problems with discontinuous Dirichlet boundary conditions, Comput. Methods Appl. Math. 18 (2018), no. 3, 373-381.

[5] L. Beirão da Veiga, F. Brezzi, A. Cangiani, G. Manzini, L. D. Marini and A. Russo, Basic principles of virtual element methods, Math. Models Methods Appl. Sci. 23 (2013), no. 1, 199-214.

[6] S. C. Brenner, A. Çeşmelioğlu, J. Cui and L.-Y. Sung, A nonconforming finite element method for an acoustic fluid-structure interaction problem, Comput. Methods Appl. Math. 18 (2018), no. 3, 383-406.

[7] C. Cancès, C. Chainais-Hillairet and S. Krell, Numerical analysis of a nonlinear free-energy diminishing duality finite volume scheme for convection diffusion equations, Comput. Methods Appl. Math. 18 (2018), no. 3, 407-432.

[8] C. Carstensen and F. Hellwig, Constants in discrete Poincaré and Friedrichs inequalities and discrete quasi-interpolation, Comput. Methods Appl. Math. 18 (2018), no. 3, 433-450. 
[9] B. Cockburn, J. Gopalakrishnan and R. Lazarov, Unified hybridization of discontinuous Galerkin, mixed, and continuous Galerkin methods for second order elliptic problems, SIAM J. Numer. Anal. 47 (2009), no. 2, 1319-1365.

[10] D. A. Di Pietro and M. Vohralik, A review of recent advances in discretization methods, a posteriori error analysis, and adaptive algorithms for numerical modeling in geosciences, Oil \& Gas Sci. Technol. Rev. IFP Energ. Nouv. 69 (2014), no. 4, 701-729.

[11] J. Droniou, R. Eymard, T. Gallouët, C. Guichard and R. Herbin, The Gradient Discretisation Method, Math. Appl., Springer, Heidelberg, 2018.

[12] A. Ern and J.-L. Guermond, Abstract nonconforming error estimates and application to boundary penalty methods for diffusion equations and time-harmonic Maxwell's equations, Comput. Methods Appl. Math. (2017), DOI 10.1515/cmam2017-0058.

[13] L. Formaggia, J.-F. Gerbeau, F. Nobile and A. Quarteroni, Numerical treatment of defective boundary conditions for the Navier-Stokes equations, SIAM J. Numer. Anal. 40 (2002), no. 1, 376-401.

[14] T. Gallouët, Discrete functional analysis tools for some evolution equations Comput. Methods Appl. Math. 18 (2018), no. 3, 477-493.

[15] J. S. Hesthaven, G. Rozza and B. Stamm, Certified Reduced Basis Methods for Parametrized Partial Differential Equations, Springer Briefs Math., Springer, Cham, 2016.

[16] J. Kreeft and M. Gerritsma, Mixed mimetic spectral element method for Stokes flow: A pointwise divergence-free solution, J. Comput. Phys. 240 (2013), 284-309.

[17] G. Morel, C. Buet and B. Després, Trefftz discontinuous Galerkin method for Friedrichs systems with linear relaxation: Application to the $P_{1}$ model, Comput. Methods Appl. Math. 18 (2018), no. 3, 521-557.

[18] R. H. Nochetto and A. Veeser, Primer of adaptive finite element methods, in: Multiscale and Adaptivity: Modeling, Numerics and Applications, Lecture Notes in Math. 2040, Springer, Heidelberg (2012), 125-225.

[19] A. Nouy, Low-rank tensor methods for model order reduction, in: Handbook of Uncertainty Quantification, Springer International Publishing, Cham (2016), 1-26. 